\title{
A REVIEW STUDY OF TRAFFIC SIGNAL VIOLATION DETECTION USING ARTIFICIAL INTELLIGENCE
}

\author{
${ }^{1}$ Dantene Davis, ${ }^{2}$ Abhishek Singh, ${ }^{3}$ Amarjeeth Singh, ${ }^{4}$ Fahad Ahmad \\ 1,2,3,4,B.E Students Department of CSE, RR Institute of Technology, Bengaluru, India
}

Article DOI: https://doi.org/10.36713/epra7139

DOI No: 10.36713/epra7139

\begin{abstract}
In the new evolving world, traffic rule violations have become a central issue for majority of the developing countries. The numbers of vehicles are increasing rapidly as well as the numbers of traffic rule violations are increasing exponentially. Managing traffic rule violations has always been a tedious and compromising task. Even though the process of traffic management has become automated, it's a very challenging problem, due to the diversity of plate formats, different scales, rotations and non-uniform illumination conditions during image acquisition. The principal objective of this project is to control the traffic rule violations accurately and cost effectively. The proposed model includes an automated system which uses IR sensors and camera based on Raspberry PI to capture video. The project presents Automatic Number Plate Recognition (ANPR) techniques and other image manipulation techniques for plate localization and character recognition which makes it faster and easier to identify the number plates. After recognizing the vehicle number from number plate, the SMS based module is used to notify the vehicle owners about their traffic rule violation. An additional SMS is sent to Regional Transport Office (RTO) for tracking the report status.
\end{abstract}

KEYWORDS- Automatic Number Plate Recognition (ANPR), Artificial Neural Network, Image acquisition, CNN, Tesseract OCR, Canny Edge Detection.

\section{INTRODUCTION}

Automation in day to day life has gained importance in recent years. The number of accidents on the roads is due to the rule violations such as breaking traffic signals, over-speeding, driving on wrong sides etc. To avoid such traffic violations, traffic police has to be present on the road and has to continuously check if some vehicle is violating the rule. A certain automated solutions were developed to eliminate the violations; however each of them had certain limitations. For example, the video capturing cameras eliminated need of a authority to be present to check rule violation. However, whole stored video had to be checked manually for the rule violation scenario. In this proposed system, a solution for signal breaking violation is given .The system includes an automated system by using IR sensor, camera and number plate recognition application. In this system IR sensor will be placed near zebra crossing line. If any vehicle crosses the zebra line, the desktop application will be initiated and will capture number plate image. Number plate recognition application by using image processing algorithm will recognize number plate and SMS will be sent to the offender in case of rule violation scenario.

Solid In all the countries there are driving rules available for people to drive carefully by being honest and respecting. When these rules are broken it is defined as a road violation. There road violations that happens in day to day traffic. For an example most common violations such as red light violation, over-speeding, overtaking other vehicles through double white lines and single white lines. These road protocols are applied thoroughly for the places where most of the accidents happen. Research team have implemented an OpenCV and python based system upon a PC. This system is implemented to ease the work to the police using a user interface. Image processing technology is used to detect the lanes, 


\section{SJIF Impact Factor 2021: 8.013| ISI I.F.Value:1.241| Journal DOI: 10.36713/epra2016 ISSN: 2455-7838(Online) EPRA International Journal of Research and Development (IJRD)}

vehicles and will identify vehicles who break rules. The team have implemented the system with the ultrasonic sensors in order to system to receive conditions to identify when a violation occur. It will be easier for the police to catch who break laws of the traffic and for the policemen who take bribes and dishonest people will also be reduced. An image of the violation with the location, time, date and an image of the vehicle to the nearest policemen devices by deriving devices around the current location that violation happened.

\{This paper is structured as follows: Section II offers a thorough overview of the various violation systems introduced and algorithms used in various publications and research projects. Section III offers methodology, section IV offers experimental results and section $\mathrm{V}$ offers results. The conclusion and future work are presented in Section VI.\}

\section{RELATED WORK}

The number of new vehicles on the road is increasing rapidly, which in turn causes highly congested roads and serving as a reason to break traffic rules by violating them. This leads to a high number of road accidents. Traffic violation detection systems using computer vision are a very efficient tool to reduce traffic violations by tracking and
Penalizing. The proposed system by Ruben J Franklin, Mohana was implemented using YOLOV3 object detection for traffic violation detections such as signal jump, vehicle speed, and the number of vehicles. Further, the system is optimized in terms of accuracy. Using the Region of interest and location of the vehicle in the duration of frames, determining signal jump. This implementation obtained an accuracy of $97.67 \%$ for vehicle count detection and an accuracy of $89.24 \%$ for speed violation detection. the proposed architecture of surveillance system with intelligent detection and tracking of multiple vehicles from the surveillance input video using YOLOv3 as an object detection algorithm. This is done through a neural network and an object detection model which are used in the classification of the moving objects into different respective classes, thus achieving vehicle classification. Next, from the same given video footage, traffic lights, zebra crossing, different lanes, and traffic signs are classified this comes under environment awareness. Combining these two, now violations are detected based on violations are then detected these can occur on the road which are signal jump, speed detection, and vehicle count. The main objective is to detect multiple vehicle violation detections and it gives a more detailed picture of concepts and technology involved in creating a traffic violation detection system using computer vision.

\section{METHODOLOGY}

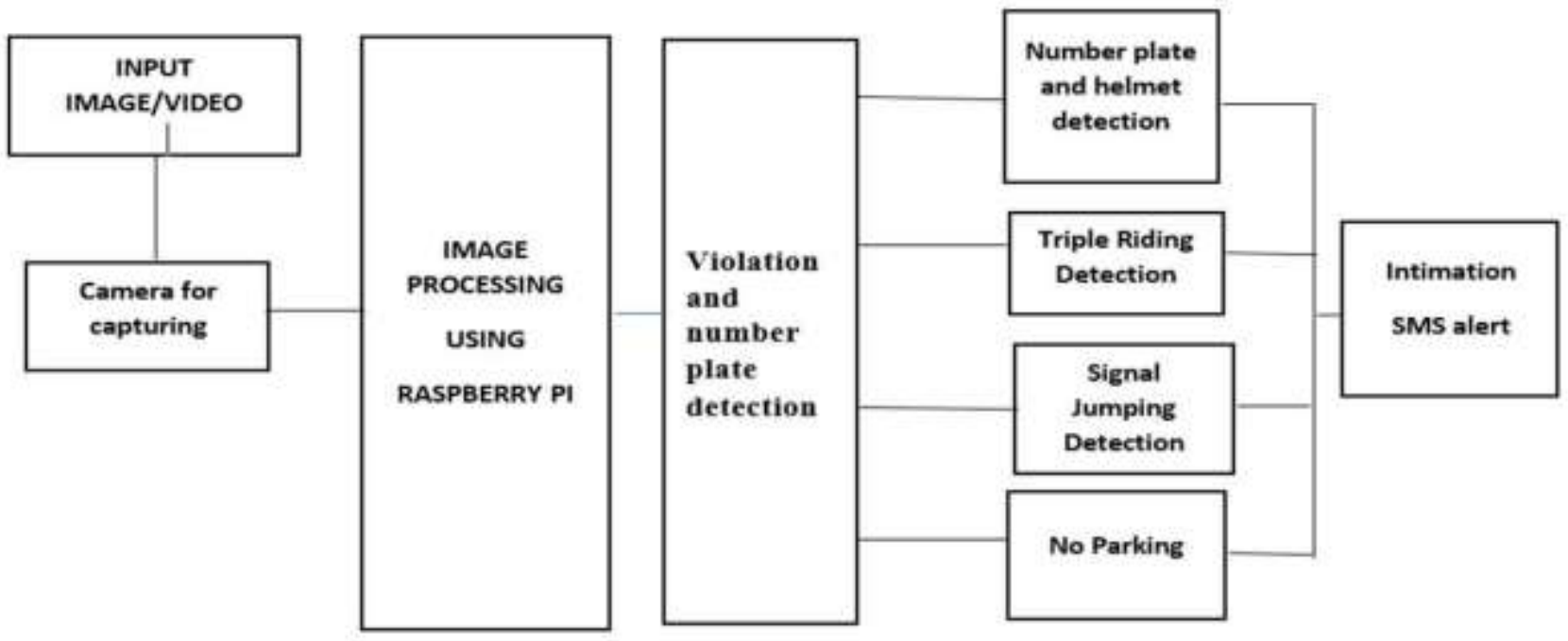

Fig 3.1 : System Architecture of Traffic Intrusion System

License plate recognition (LPR) is one form of ITS (Intelligent Transport System) technology that not only recognizes and counts the number of vehicles but also differentiates them. For some applications, such as electronic toll collection and red-light violation enforcement, LPR records license plates alphanumerically so the vehicle owner can be assessed the appropriate amount of fine. In others cases, like commercial vehicle operations or secureaccess control, a vehicle's license plate is compared against a database of acceptable ones to determine whether a truck can bypass a weigh station or a car can enter a gated community or parking lot. A license plate is the unique identification of a vehicle. The basic issues in real-time license plate recognition are the accuracy and the recognition speed. License Plate 


\section{SJIF Impact Factor 2021: 8.013| ISI I.F.Value:1.241| Journal DOI: 10.36713/epra2016 ISSN: 2455-7838(Online) EPRA International Journal of Research and Development (IJRD)}

Volume: 6 | Issue: 5 | May 2021

- Peer Reviewed Journal

Recognition (LPR) has been applied in numerous applications such as automatically identifying vehicles in parking lots, access control in a restricted area and detecting and verifying stolen vehicles. Quality of algorithms used in a license plate detector determines the speed and accuracy of the license plate detection. In the past, a number of techniques have been proposed for locating the plate through visual image processing.
A video is taken from a camera, and then each frame of the video is processed as the image. In this stage the license plate region from the given image is located and isolated. Quality of the image plays an important part hence prior to this stage preprocessing of the image is necessary. So first each frame pre-processed by binarization, noise reduction and edge detection. Then, the license plate is located by different image processing technique.

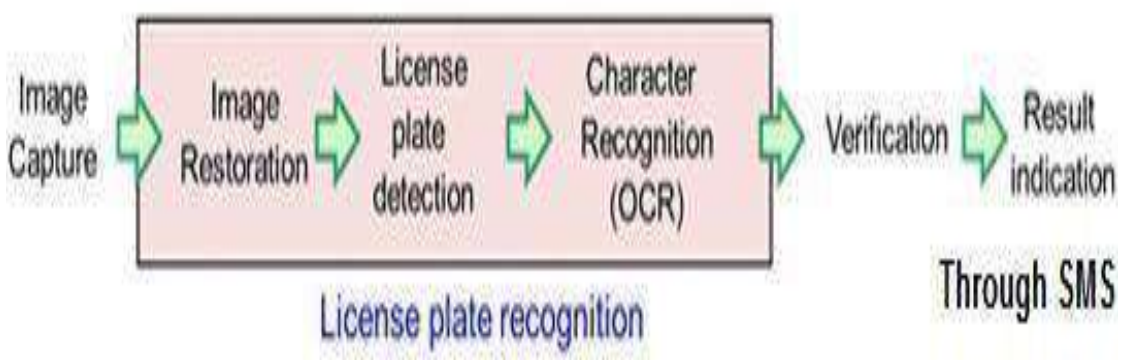

Fig 3.2 : Analysis Model of Traffic Intrusion System

Tesseract OCR: The number plate recognition using Tesseract OCR starts with image acquisition, then the image is being pre-processed to remove any distortions and noise from the image. Then number plate is then localized irrelevant part and characters in the number plate are segmented and then the recognized and output. The license plate recognition detects number plates of all types of vehicles like car, truck and motorcycle. It also works on different number plate styles and fonts with high accuracy.

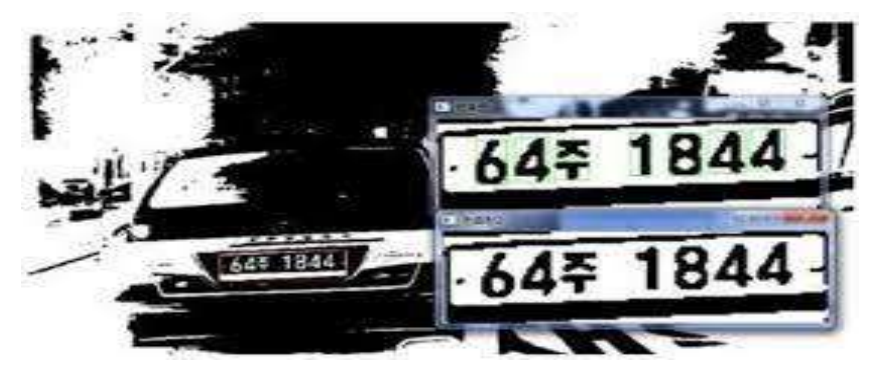

Fig 3.3 : Number Plate Detection using Tesseract OCR

Gaussian Blur method: Gray scaling and blurring: As the part of pre-processing the input frame got

from the CCTV footage, the image is Gray scaled and blurred with Gaussian Blur method.

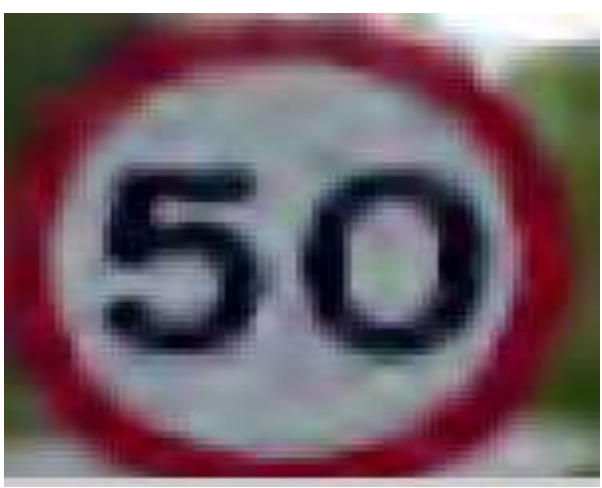

Fig.3.4: Image Segmentation 


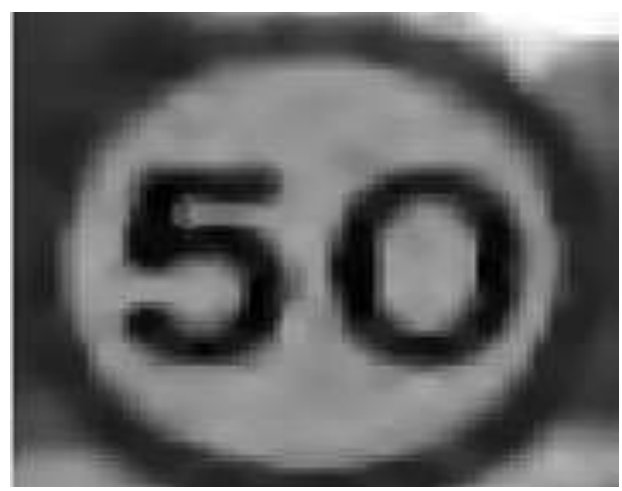

Fig. 3.5: Gray Scaling

Canny Edge Detection: The canny edge detector is an multi stage algorithm is used to detect wide range of edges in image. Edge detection is the process of identifying the edge in a digital image where the intensity of the image changes sharply or has discontinuities. The unwanted region is removed other than the number plate section.

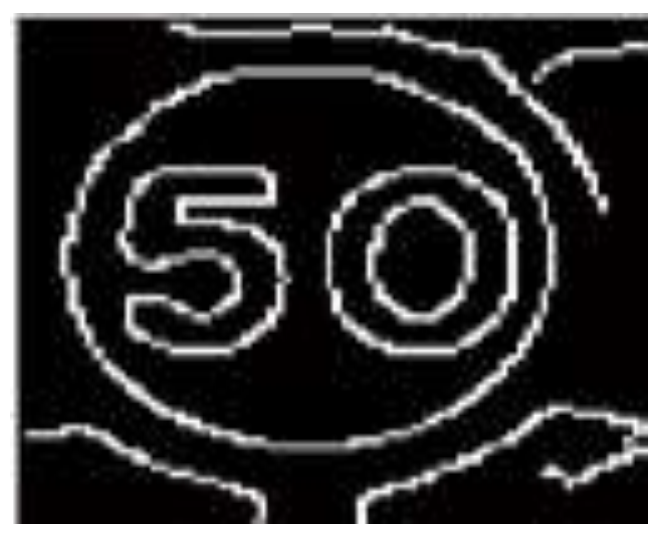

Fig. 3.6: Canny Edge Image

through CNN and an object detection model which

CNN (Convolutional neural network) :The proposed architecture of surveillance system with intelligent detection and tracking of multiple vehicles from the surveillance input video. This is done are used in the classification of the moving objects into different respective classes, thus achieving vehicle classification.

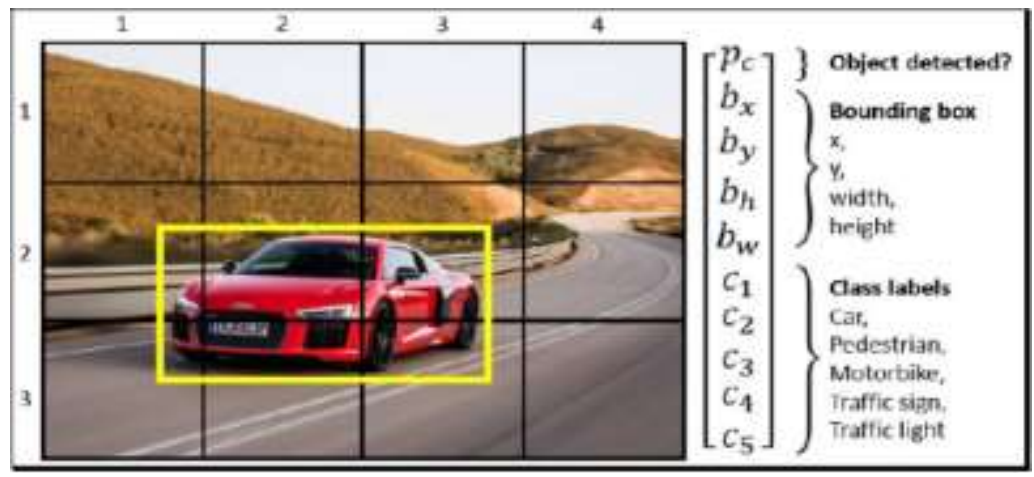

Fig. 3.7: Object Detection 


\section{EPRA International Journal of Research and Development (IJRD)}

\section{EXPERIMENTAL RESULT}

MOTION DETECTION OPERATION: When there is motion, the differences of the profiles are larger than the case when there is no motion. The motion can be detected by selecting a threshold value.
VEHICLE DETECTION ALGORITHM: A vehicle detection operation is applied on the profile of the unprocessed image. The method used here is based on applying edge detector operators to a profile of the image edges are less sensitive to the variation of ambient lighting and are used in full frame applications (detection).

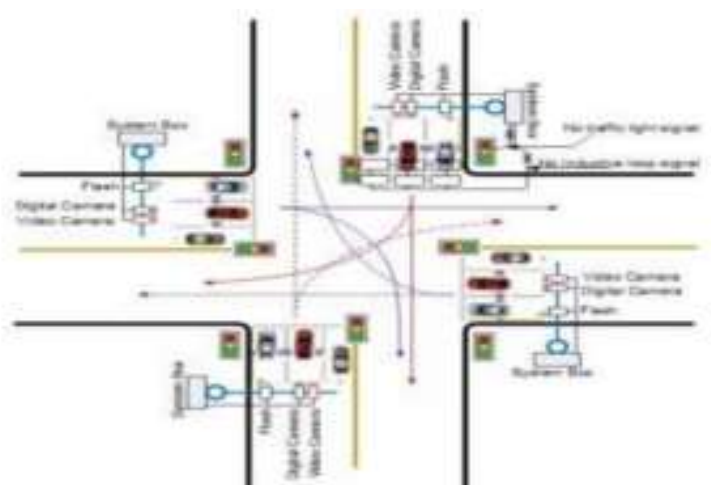

Fig. 4.1: An overview of a purely video processing

TRAFFIC MOVEMENTS AT JUNCTIONS (TMJ): The first step to measure the TMJ parameters using the key region method is to cover the boundary of the junction by a polygon in such a way that all the entry and exit paths of the junction cross the polygon. However, the polygon should not cover the pedestrian marked lines. This step is shown in the figure given below. The second step of the algorithm is to define a minimum number of key regions inside the boundary of the polygon, covering the junction.

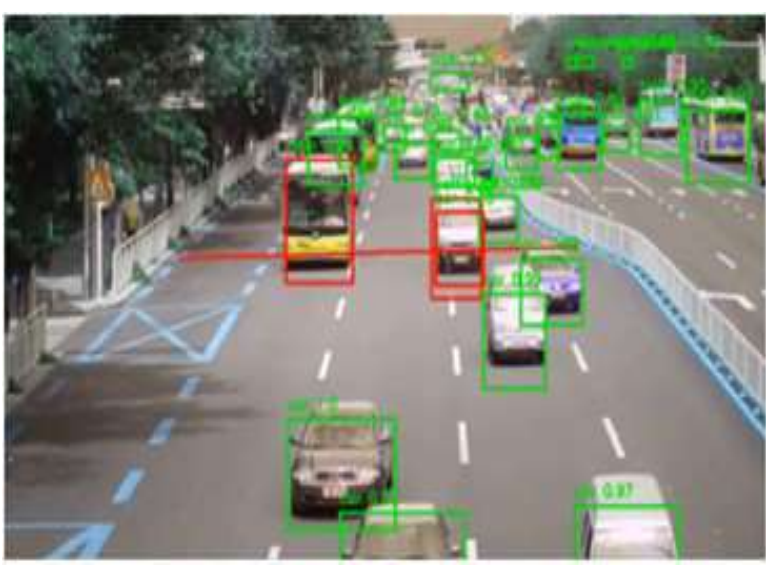

Fig. 4.2: Detection of a traffic violation output frame: bounding box and violation detection (signal jump) 


\section{SJIF Impact Factor 2021: 8.013| ISI I.F.Value:1.241| Journal DOI: 10.36713/epra2016 ISSN: 2455-7838(Online) EPRA International Journal of Research and Development (IJRD)}

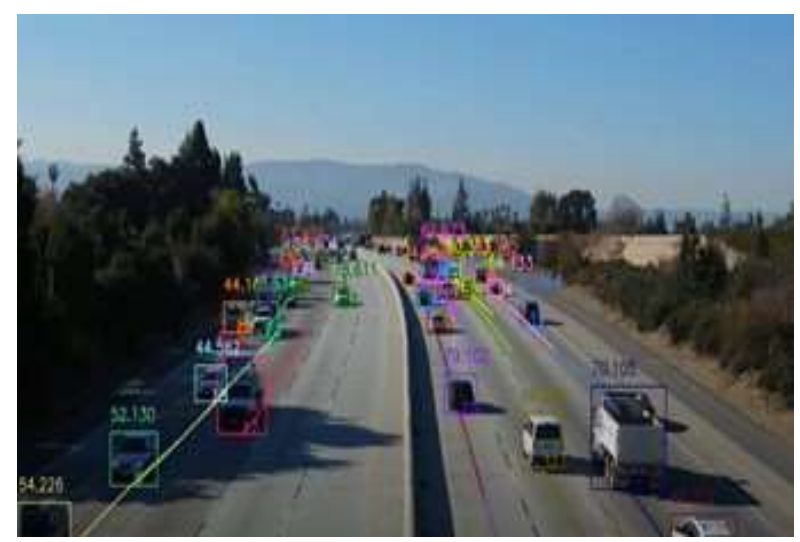

Fig. 4.3: Speed Violation and Lane Change Detection

\section{RESULT}

Through this paper Number Plate of the vehicle is authenticated and data is taken. Since this model is capable of detecting vehicles in every frame it is having been enhanced to detect the speed of the vehicle in a video. Detection of vehicle crossing during a red light in the image and locate the exact location. Data of the vehicle is taken when the people riding on two-wheeler is more than two. Vehicle parked in the no parking area is given five minutes time to change the vehicle from the spot if not changed violation is detected and fine is sent through SMS. The speed is calculated using all the three principles, the rate of change of the object is the video frame by frame gives us the speed but it is not the true speed. This because the object in the video doesn't move linearly hence the area of the boundary frame by frame gives us the actual distance travelled by the vehicle in the video. The anchor points add more perception to the parameters. With this parameter, the speed of the vehicle is determined.

\section{CONCLUSION}

Detections of traffic violation in the video surveillance is challenging as the number of vehicles on the road and traffic rules are depended on the different area of the road and timings. Here in this paper, the methods for traffic surveillance have been presented and the work on motion detection, license plate extraction and character recognition is carried out. Results show that the detection of multiple traffic violations from a single input source is archivable. It should be noted that robust motion detection is a critical task and its performance is affected by the presence of varying illumination, background motion, camouflage, shadow, and etc. The system has an accuracy of $97.67 \%$ for vehicle count detection and an accuracy of $89.24 \%$ to detect the vehicle speed. The detection time is lower for high dense traffic flow. Thus, the system operation speed is dependent on the density of traffic.

\section{REFERENCES}

1. "Implementing Lane Departure Warning System on Raspberry Pi”, University Tunku Abdul Rahman, May 2016

2. "A Real-Time Vision System for Night time Vehicle Detection and Traffic Surveillance", IEEE transactions on industrial electronics, Volume-58, No-5, May 2011.

3. "Morphological Image Processing Approach of Vehicle Detection for Real-Time Traffic Analysis", Science International Journal of Computer Science and Engineering, Volume-2, Issue-5, May 2014.

4. "Development of an automated Red Light Violation Detection System (RLVDS) for Indian vehicles", IEEE National Conference on Computing and Communication Systems, COCOSYS-09, CS11 [Accessed: 14-JULY-2016]

5. "Vehicle Detection under Day and Night Illumination", Proc. of ISCS-IIA99, May 2016.

6. "A real-time computer vision system for vehicle tracking and traffic surveillance", Transportation Research Part C 6, CA 94720-1776, USA, Dec 1998.

7. "Automated Driving Vehicle Using Image Processing", International Journal of Computer Science and Engineering, Volume-2, Issue-4, April 2014.

8. Handaric R. et al. "An Image Recognition Software Tool for Car Identification Based on License Plate", Transylvania University of Braşov Series I: Engineering Sciences, Volume-5 (54) No. 1, 2012.

9. "Object Detection in Image Processing Using Edge Detection Techniques", and IOSR Journal of Engineering, ISSN (e): 2250-3021, ISSN (p): 22788719 Vol. 04, Issue 03, March 2014.

10. "Hybrid Parking Detection", United States Patent Application Publication, Patent No. US20150117705A1, April 2015. 